\title{
The degree of mutual anisotropy of biological liquids polycrystalline nets as a parameter in diagnostics and differentiations of hominal inflammatory processes
}

\author{
O.V. Angelsky ${ }^{a}$, Yu.A. Ushenko ${ }^{a}$, V.O. Balanetska*a \\ ${ }^{\mathrm{a} C}$ Correlation Optics Department, Chernivtsi National University, 2 Kotsyubinsky Str., Chernivtsi, \\ 58012, Ukraine; \\ ${ }^{\mathrm{b}}$ Optics and Spectroscopy Department, Chernivtsi National University, 2 Kotsyubinsky Str., \\ Chernivtsi, 58012, Ukraine
}

\begin{abstract}
To characterize the degree of consistency of parameters of the optically uniaxial birefringent protein nets of blood plasma a new parameter - complex degree of mutual anisotropy is suggested. The technique of polarization measuring the coordinate distributions of the complex degree of mutual anisotropy of blood plasma is developed. It is shown that statistic approach to the analysis of the complex degree of mutual anisotropy distributions of blood plasma is effective during the diagnostics and differentiation of an acute inflammatory processes as well as acute and gangrenous appendicitis.
\end{abstract}

Keywords: Polarization, birefringence, anisotropy, statistic moments, blood plasma

\section{INTRODUCTION}

Historically, the optical methods of biological object diagnostics may be divided into three groups:

- The spectrophotometric methods ${ }^{1-3}$, which are based on analysis of spatial $(r)$ or time $(\tau)$ changes of radiation field intensity, scattered by biological tissues (BT);

- Polarization methods are based on usage of coherency matrix of complex amplitude $\{\mathrm{K}(r, \tau)\}^{4-6}$

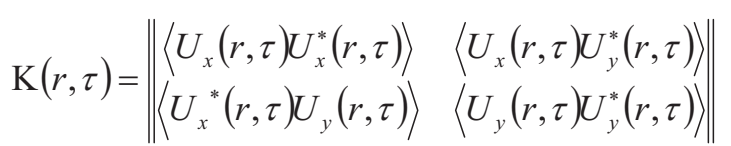

and the analysis of the degree of polarization $P(r)$ as the factor of correlation of complex orthogonal components of the electromagnetic fluctuation $U_{x}, U_{y}$ at one of the points $(r)$ of the scattered radiation field ${ }^{7}$

$$
P(r)=\sqrt{1-\frac{\left.4 \mid\left\langle U_{x}(r, \tau) U_{x}^{*}(r, \tau)\right\rangle\left\langle U_{y}(r, \tau) U_{y}^{*}(r, \tau)\right\rangle-\left\langle U_{x}(r, \tau) U_{y}^{*}(r, \tau)\right\rangle\left\langle U_{y}(r, \tau) U_{x}^{*}(r, \tau)\right\rangle\right]}{\left[\left\langle U_{x}(r, \tau) U_{x}^{*}(r, \tau)\right\rangle+\left\langle U_{y}(r, \tau) U_{y}^{*}(r, \tau)\right\rangle\right]^{2}} ;}
$$

- The correlation methods which are based on the analysis of the correlation degree $J$ of the parallel polarization

*yuriyu@gmail.com

Tenth International Conference on Correlation Optics, edited by Oleg V. Angelsky, Proc. of SPIE Vol. 8338, 83380S - (c) 2011 SPIE · CCC code: 0277-786X/11/\$18 - doi: 10.1117/12.920065 
components $U_{x}\left(r_{1}\right), U_{x}\left(r_{2}\right)$ of the light fluctuation at different points of object field $\left(r_{1}, r_{2}\right)^{7-8}$

$$
J=\frac{\left\langle U_{x}\left(r_{1}, \tau\right) U_{x}^{*}\left(r_{2}, \tau\right)\right\rangle-\left\langle U_{x}^{*}\left(r_{1}, \tau\right) U_{x}\left(r_{2}, \tau\right)\right\rangle}{\left\langle U_{x}\left(r_{1}, \tau\right) U_{x}^{*}\left(r_{2}, \tau\right)\right\rangle+\left\langle U_{x}^{*}\left(r_{1}, \tau\right) U_{x}\left(r_{2}, \tau\right)\right\rangle}
$$

For the complex analysis of polarizationally heterogeneous laser radiation fields a new approach was suggested in ${ }^{9-15}$, based on the generalization of coherence matrix $\{\mathrm{K}(r, \tau)\}$ by the polarization coherence matrix $\left\{\Phi\left(r_{1}, r_{2}, \tau\right)\right\}$ for two points $\left(r_{1}, r_{2}\right)$

$$
\{\mathrm{K}(r, \tau)\} \Rightarrow\left\{\Phi\left(r_{1}, r_{2}, \tau\right)\right\}
$$

In expanded form (4) can be rewritten as follows

$$
\left\|\begin{array}{ll}
\left\langle U_{x}(r, \tau) U_{x}^{*}(r, \tau)\right\rangle & \left\langle U_{x}(r, \tau) U_{y}^{*}(r, \tau)\right\rangle \\
\left\langle U_{y}(r, \tau) U_{x}^{*}(r, \tau)\right\rangle & \left\langle U_{y}(r, \tau) U_{y}^{*}(r, \tau)\right\rangle
\end{array}\right\| \Rightarrow\left\|\begin{array}{lll}
\left\langle U_{x}\left(r_{1}, \tau\right) U_{x}^{*}\left(r_{2}, \tau\right)\right\rangle & \left\langle U_{x}\left(r_{1}, \tau\right) U_{y}^{*}\left(r_{2}, \tau\right)\right\rangle \\
\left\langle U_{y}\left(r_{1}, \tau\right) U_{x}^{*}\left(r_{2}, \tau\right)\right\rangle & \left\langle U_{y}\left(r_{1}, \tau\right) U_{y}^{*}\left(r_{2}, \tau\right)\right\rangle
\end{array}\right\| .
$$

Here \langle\rangle means the averaging operation over the time $\tau$ of orthogonal components $U_{x}, U_{y}$ of electromagnetic wave complex amplitude at the points with coordinates $\left(r_{1}, r_{2}\right)$.

$\mathrm{In}^{16}$ for characterizing the consistency between the polarization states the object field in the points $\left(r_{1}, r_{2}\right)$ with the intensities $I\left(r_{1}, \tau\right), I\left(r_{2}, \tau\right)$ a new parameter - complex degree of mutual polarization (CDMP) $V\left(r_{1}, r_{2}, \tau\right)-$ is introduced. It has the following analytical form:

$$
V\left(r_{1}, r_{2}, \tau\right)=4 \frac{v_{1}^{2}+v_{2}^{2}+v_{3}^{2}}{I\left(r_{1}, \tau\right) I\left(r_{2}, \tau\right)}
$$

where $v_{i}$ - the coefficients that are formed by combinations of the elements of the polarization coherency matrix $\left\{\Phi\left(r_{1}, r_{2}, \tau\right)\right\}$

$$
\begin{aligned}
& v_{1}=\frac{\left\langle U_{x}\left(r_{1}, \tau\right) U_{x}^{*}\left(r_{2}, \tau\right)\right\rangle-\left\langle U_{y}\left(r_{1}, \tau\right) U_{y}^{*}\left(r_{2}, \tau\right)\right\rangle}{2}, \\
& v_{2}=\frac{\left\langle U_{x}\left(r_{1}, \tau\right) U_{y}^{*}\left(r_{2}, \tau\right)\right\rangle+\left\langle U_{y}\left(r_{1}, \tau\right) U_{x}^{*}\left(r_{2}, \tau\right)\right\rangle}{2}, \\
& v_{3}=i \frac{\left\langle U_{x}\left(r_{1}, \tau\right) U_{y}^{*}\left(r_{2}, \tau\right)\right\rangle-\left\langle U_{y}\left(r_{1}, \tau\right) U_{x}^{*}\left(r_{2}, \tau\right)\right\rangle}{2} .
\end{aligned}
$$

These elements are determined as the difference of the values of visibility of interference images formed by electromagnetic waves from the points $r_{1}, r_{2}$. A measurement of these parameters is made for different orientations of the polarization filter placed before the photodetector. In this case, the value $v_{1}$ is determined by the difference of 
contrast values for the rotation angles of the analyzer transmission plane $0^{\circ}, 90^{\circ}$ to the incidence plane; $v_{2}-$ for angles $45^{\circ}, 135^{\circ} ; v_{3}$ - for the rotation of the axis of maximum velocity of quarter-wave plates at angle $+45^{\circ}$ (right circulation) and $-45^{\circ}$ (right circulation).

It is easy to show that the expression of the complex degree of mutual polarization CDMP in the case of laser radiation scattered stationary field takes the next form

$$
V\left(r_{1}, r_{2}\right)=\frac{\left(U_{x}\left(r_{1}\right) U^{*}{ }_{x}\left(r_{2}\right)+U_{y}\left(r_{1}\right) U^{*}{ }_{y}\left(r_{2}\right)\right)^{2}}{I\left(r_{1}\right) I\left(r_{2}\right)}
$$

This "two-point" theoretical approach ((4) - (8)) was extended to the analysis of polarization-inhomogeneous laser images of human BT with the aim of experimental diagnostics of optical anisotropic structure. $\operatorname{In}^{17}$ a method of direct polarization measurement of the real part of CDMP for different points $\left(r_{1}, r_{2}\right)$ of optically thin image (attenuation coefficient $\tau \leq 0,1$ ) of BT layers is proposed

$$
\operatorname{Re}\{V\} \equiv \widetilde{V}\left(r_{1}, r_{2}\right)=\frac{\left\{U_{x}\left(r_{1}\right) U_{x}\left(r_{2}\right)-U_{y}\left(r_{1}\right) U_{y}\left(r_{2}\right) \cos \left[\varphi\left(r_{1}\right)-\varphi\left(r_{2}\right)\right]\right\}^{2}}{I\left(r_{1}\right) I\left(r_{2}\right)},
$$

were $\varphi\left(r_{1}\right)$ и $\varphi\left(r_{2}\right)$ - the phase shifts between the orthogonal components $U_{x}, U_{y}$ of laser beam amplitude.

The ranges of changes of the $1^{\text {st }}-4^{\text {th }}$ distribution order statistic moments $\widetilde{V}(x, y)$ of the corresponding laser images, important for diagnostics of the human connective tissue oncologic state were determined $\mathrm{in}^{17}$. On the other hand, such analysis techniques of $\widetilde{V}(x, y)$ lead to disregarding the BT extracellular matrix birefringence, which is a principal physical mechanism of their polarizationally-heterogeneous images formation. That is why it appears to be important to search for new diagnostic parameters directly characterizing the degree of consistency of optical axes and birefringence orientations of various points of protein fibrils network forming the BT extracellular matrix. From the other side the birefringent polycrystalline protein nets of various biological fluids (blood, bile, urine, etc.) are more obtainable for diagnostic tasks of human physiological state.

This work is aimed on substantiating the technique of polarization-correlation mapping, on the basis of a new parameter measurement, which characterizes the mutual anisotropy of the birefringent networks of a human blood plasma layers (smears) for physiological and pathological state diagnostics.

\section{THEORETICAL ANALYSIS OF LASER RADIATION PARAMETERS TRANSFORMATION BY THE NETWORK OF OPTICALLY UNIAXIAL BIREFRINGENT PROTEIN CRYSTALS}

As a base for modeling the optical properties of blood plasma we use the conception of anisotropy observed in BT protein networks developed in ${ }^{17}$ :

- blood plasma can be considered as a two-component amorphous-crystalline structure;

- optically anisotropic - liquid-crystalline phase consisting of a set of optically uniaxial birefringent liquid crystals of various types: amino acids of albumin and globulin.

The optical properties of amorphous $\{A\}$ and crystalline $\{C\}$ components of biological liquids can be exhaustively described using the following Jones operators ${ }^{17}$ 


$$
\begin{gathered}
\{A\}=\left\|\begin{array}{ll}
a_{11} & a_{12} \\
a_{21} & a_{22}
\end{array}\right\|=\left\|\begin{array}{cc}
\exp (-\tau l) & 0 \\
0 & \exp (-\tau l)
\end{array}\right\| ; \\
\{D\}=\left\|\begin{array}{ll}
d_{11} & d_{12} \\
d_{21} & d_{22}
\end{array}\right\|=\left\|\begin{array}{ll}
\cos ^{2} \rho+\sin ^{2} \rho \exp (-i \delta) ; & \cos \rho \sin \rho[1-\exp (-i \delta)] ; \\
\cos \rho \sin \rho[1-\exp (-i \delta)] & \sin ^{2} \rho+\cos ^{2} \rho \exp (-i \delta) ;
\end{array}\right\| .
\end{gathered}
$$

Here, $\tau$ is the absorption coefficient for laser radiation in the biological liquid layer with the geometric thickness $l ; \rho$ - direction of the optical axis; $\delta=2 \pi / \lambda \Delta n d$ - phase shift between the orthogonal components $E_{x}$ and $E_{y}$ of the amplitude of illuminating laser light with the wavelength $\lambda ; \Delta n$ - index of birefringence.

The Jones matrix of the biological liquid layer, where isotropic and anisotropic creations lie in one plane, can be expressed as a sum of operators $\{A\}$ and $\{C\}$

$$
\{M\}=\{A\}+\{C\}=\left\|\begin{array}{ll}
a_{11}+c_{11} ; & a_{12}+c_{12} ; \\
a_{21}+c_{21} ; & a_{22}+c_{22}
\end{array}\right\|,
$$

Analytically, the process of transformation of the amplitude and phase of the laser beam by the crystal protein of blood plasma at coordinates $r$ can be described by the equation

$$
\begin{aligned}
& \left(\begin{array}{l}
U_{x}(r) \\
U_{y}(r)
\end{array}\right)=\left\|\begin{array}{ll}
d_{11}(r) & d_{12}(r) \\
d_{21}(r) & d_{22}(r)
\end{array}\right\|\left(\begin{array}{c}
U_{0 x} \\
U_{0 y} \exp \left(-i \delta_{0}\right)
\end{array}\right)= \\
& =\left(\begin{array}{c}
d_{11}(r) U_{0 x}+d_{12}(r) U_{0 y} \exp \left(-i \delta_{0}\right) \\
d_{21}(r) U_{0 x}+d_{22}(r) U_{0 y} \exp \left(-i \delta_{0}\right)
\end{array}\right)=\left(\begin{array}{c}
d_{11}(r)+d_{12}(r) \operatorname{tg} \Omega_{0} \exp \left(-i \delta_{0}\right) \\
d_{21}(r)+d_{22}(r) \operatorname{tg} \Omega_{0} \exp \left(-i \delta_{0}\right)
\end{array}\right) .
\end{aligned}
$$

Here $\delta_{0}$ - the phase shift between the orthogonal components $U_{0 x}$ and $U_{0 y}$ of the illuminating laser beam amplitude, $\operatorname{tg} \Omega_{0}=\frac{U_{0 y}}{U_{0 x}}$.

In accordance with the approach outlined in ${ }^{17}$, for a two-point $\left(r_{1}, r_{2}\right)$ generalization of the analytical description of the anisotropy parameters $\left(\rho_{1}\left(r_{1}\right), \rho_{2}\left(r_{2}\right)\right.$ and $\left.\delta_{1}\left(r_{1}\right), \delta_{2}\left(r_{2}\right)\right)$ of protein crystals network of blood plasma use (6), (7), taking into account equation (13)

$$
\begin{gathered}
v_{1}\left(d_{i k}\right)=\frac{\left[d_{11}\left(r_{1}\right)+d_{12}\left(r_{1}\right) \operatorname{tg} \Omega_{0} \exp \left(-i \delta_{0}\right)\right]\left[d_{11}\left(r_{2}\right)+d_{12}\left(r_{2}\right) \operatorname{tg} \Omega_{0} \exp \left(-i \delta_{0}\right)\right]^{*}}{2}- \\
-\frac{\left[d_{21}\left(r_{1}\right)+d_{22}\left(r_{1}\right) \operatorname{tg} \Omega_{0} \exp \left(-i \delta_{0}\right)\right]\left[d_{21}\left(r_{2}\right)+d_{22}\left(r_{2}\right) \operatorname{tg} \Omega_{0} \exp \left(-i \delta_{0}\right)\right]^{*}}{2}, \\
v_{2}\left(d_{i k}\right)=\frac{\left[d_{11}\left(r_{1}\right)+d_{12}\left(r_{1}\right) \operatorname{tg} \Omega_{0} \exp \left(-i \delta_{0}\right)\right]\left[d_{21}\left(r_{2}\right)+d_{22}\left(r_{2}\right) \operatorname{tg} \Omega_{0} \exp \left(-i \delta_{0}\right)\right]^{*}}{2}+ \\
+\frac{\left[d_{21}\left(r_{1}\right)+d_{22}\left(r_{1}\right) \operatorname{tg} \Omega_{0} \exp \left(-i \delta_{0}\right)\right]\left[d_{11}\left(r_{2}\right)+d_{12}\left(r_{2}\right) \operatorname{tg} \Omega_{0} \exp \left(-i \delta_{0}\right)\right]^{*}}{2} \\
v_{3}\left(d_{i k}\right)=i\left\{\begin{array}{l}
\frac{\left[d_{11}\left(r_{1}\right)+d_{12}\left(r_{1}\right) \operatorname{tg} \Omega_{0} \exp \left(-i \delta_{0}\right)\right]\left[d_{21}\left(r_{2}\right)+d_{22}\left(r_{2}\right) \operatorname{tg} \Omega_{0} \exp \left(-i \delta_{0}\right)\right]^{*}}{2}- \\
-\frac{\left[d_{21}\left(r_{1}\right)+d_{22}\left(r_{1}\right) \operatorname{tg} \Omega_{0} \exp \left(-i \delta_{0}\right)\right]\left[d_{11}\left(r_{2}\right)+d_{12}\left(r_{2}\right) \operatorname{tg} \Omega_{0} \exp \left(-i \delta_{0}\right)\right]^{*}}{2}
\end{array}\right\} .
\end{gathered}
$$


Substituting coefficients (14) in expression (6) we obtain the following expression for the parameter $W\left(r_{1}, r_{2}\right)$ that characterizes the degree of coordination between the orientation $\left(\rho_{1}\left(r_{1}\right), \rho_{2}\left(r_{2}\right)\right)$ - phase $\left(\delta_{1}\left(r_{1}\right) ; \delta_{2}\left(r_{2}\right)\right)$ parameters of the anisotropy of different points $\left(r_{1}, r_{2}\right)$ of birefringent protein crystals network of blood plasma

$$
\begin{aligned}
& W\left(r_{1}, r_{2}\right)=\frac{\left(A\left\{d_{i k}\left[\left(r_{1}, r_{2}\right)\right],\left[\delta_{2}\left(r_{2}\right)-\delta_{1}\left(r_{1}\right)\right], \operatorname{tg} \Omega_{0}, \delta_{0}\right\}+\right.}{C\left\{d_{i k}\left[\left(r_{1}, r_{2}\right)\right],\left[\delta_{2}\left(r_{2}\right)-\delta_{1}\left(r_{1}\right)\right], \operatorname{tg} \Omega_{0}, \delta_{0}\right\}} \\
& \frac{\left.+B\left\{d_{i k}\left[\left(r_{1}, r_{2}\right)\right],\left[\delta_{2}\left(r_{2}\right)-\delta_{1}\left(r_{1}\right)\right], \operatorname{tg} \Omega_{0}, \delta_{0}\right\}\right)^{2}}{C\left\{d_{i k}\left[\left(r_{1}, r_{2}\right)\right],\left[\delta_{2}\left(r_{2}\right)-\delta_{1}\left(r_{1}\right)\right], \operatorname{tg} \Omega_{0}, \delta_{0}\right\}}
\end{aligned}
$$

were

$$
\begin{gathered}
A=\left[d_{11}\left(r_{1}\right)+d_{12}\left(r_{1}\right) \operatorname{tg} \Omega_{0} \exp \left(-i \delta_{0}\right)\right] \times \\
{\left[d_{11}\left(r_{2}\right)+d_{12}\left(r_{2}\right) \operatorname{tg} \Omega_{0} \exp \left(-i \delta_{0}\right)\right]^{*},} \\
B=\left\{\left[d_{21}\left(r_{1}\right)+d_{22}\left(r_{1}\right) \operatorname{tg} \Omega_{0} \exp \left(-i \delta_{0}\right)\right]\left[d_{21}\left(r_{2}\right)+d_{22}\left(r_{2}\right) \operatorname{tg} \Omega_{0} \exp \left(-i \delta_{0}\right)\right]^{*}\right\} \times \\
\times \exp \left\{-i\left[\delta_{2}\left(r_{2}\right)-\delta_{1}\left(r_{1}\right)\right]\right\}, \\
C=\left\{\begin{array}{l}
{\left[d_{11}\left(r_{1}\right)+d_{12}\left(r_{1}\right) \operatorname{tg} \Omega_{0} \exp \left(-i \delta_{0}\right)\right]\left[d_{11}\left(r_{1}\right)+d_{12}\left(r_{1}\right) \operatorname{tg} \Omega_{0} \exp \left(-i \delta_{0}\right)\right]^{*}+} \\
+\left[d_{21}\left(r_{1}\right)+d_{22}\left(r_{1}\right) \operatorname{tg} \Omega_{0} \exp \left(-i \delta_{0}\right)\right]\left[d_{21}\left(r_{1}\right)+d_{22}\left(r_{1}\right) \operatorname{tg} \Omega_{0} \exp \left(-i \delta_{0}\right)\right]^{*}
\end{array}\right\}+ \\
+\left\{\begin{array}{l}
{\left[d_{11}\left(r_{2}\right)+d_{12}\left(r_{2}\right) \operatorname{tg} \Omega_{0} \exp \left(-i \delta_{0}\right)\right]\left[d_{11}\left(r_{2}\right)+d_{12}\left(r_{2}\right) \operatorname{tg} \Omega_{0} \exp \left(-i \delta_{0}\right)\right]^{*}+} \\
\left.+\left[d_{21}\left(r_{2}\right)+d_{22}\left(r_{2}\right) \operatorname{tg} \Omega_{0} \exp \left(-i \delta_{0}\right)\right]\left[d_{21}\left(r_{2}\right)+d_{22}\left(r_{2}\right) \operatorname{tg} \Omega_{0} \exp \left(-i \delta_{0}\right)\right]^{*}\right\}
\end{array}\right.
\end{gathered}
$$

Further, similarly to ${ }^{17}$ we shall call parameter $W\left(r_{1}, r_{2}\right)$ the complex degree of mutual anisotropy (CDMA) of blood plasma.

Analysis of equations (13-18) shows the dependence of orthogonal components $U_{x}(r), U_{y}(r)$ of the complex amplitude on rotation of the polarization plane $\left(\Omega_{0}\right)$ of the illuminating laser wave or rotation $(\gamma)$ of blood plasma layer to the irradiation direction. The comparative diagnostic analysis of CDMA of blood plasma according to it becomes more difficult. The only exception is a case of irradiation of blood plasma by the circular polarized laser wave $U_{0}$

$$
U_{0}=\left(\begin{array}{l}
U_{0 x} \\
U_{0 y}
\end{array}\right) \equiv \frac{1}{\sqrt{2}}\left(\begin{array}{l}
1 \\
i
\end{array}\right)
$$

Therefore, for determination the analytical expression for CDMA $W\left(r_{1}, r_{2}\right)$ of two points $\left(r_{1}, r_{2}\right)$ of blood plasma we need use the relations (5), (7) and take into account the equations (8), (9)

$$
W\left(r_{1}, r_{2}\right)=\frac{\left\{\left[d_{11}\left(r_{1}\right)+i d_{12}\left(r_{1}\right)\right]\left[d_{11}\left(r_{2}\right)+i d_{12}\left(r_{2}\right)\right]^{*}+\left[d_{21}\left(r_{1}\right)+i d_{22}\left(r_{1}\right)\right]\left[d_{21}\left(r_{2}\right)+i d_{22}\left(r_{2}\right)\right]^{*}\right\}^{2}}{I\left(r_{1}\right) I\left(r_{2}\right)} .
$$


Here $I\left(r_{1}\right)$ and $I\left(r_{2}\right)$ are determined by relations

$$
\left.\begin{array}{l}
I\left(r_{1}\right)=\left\{\left[d_{11}\left(r_{1}\right)+i d_{12}\left(r_{1}\right)\right]\left[d_{11}\left(r_{1}\right)+i d_{12}\left(r_{1}\right)\right]^{*}+\left[d_{21}\left(r_{1}\right)+i d_{22}\left(r_{1}\right)\right]\left[d_{21}\left(r_{1}\right)+i d_{22}\left(r_{1}\right)\right]^{*}\right\}, \\
I\left(r_{2}\right)=\left\{\left[d_{11}\left(r_{2}\right)+i d_{12}\left(r_{2}\right)\right]\left[d_{11}\left(r_{2}\right)+i d_{12}\left(r_{2}\right)\right]^{*}+\left[d_{21}\left(r_{2}\right)+i d_{22}\left(r_{2}\right)\right]\left[d_{21}\left(r_{1}\right)+i d_{22}\left(r_{2}\right)\right]\right.
\end{array}\right\} .
$$

Taking the real part $\left(\operatorname{Re}\left\{d_{i k}(r)\right\} \equiv \widetilde{d}_{i k}(r)\right)$ of the Jones matrix elements (equation 10), we obtain an expression for the real part of the CDMA $\left(\operatorname{Re}\left\{W\left(r_{1}, r_{2}\right)\right\} \equiv \widetilde{W}\left(r_{1}, r_{2}\right)\right.$ )of blood plasma layer, which can be obtained by direct polarization measurements

$$
\widetilde{W}\left(r_{1}, r_{2}\right)=\frac{\left\{\left[\widetilde{d}_{11}\left(r_{1}\right) \widetilde{d}_{11}\left(r_{2}\right)-\widetilde{d}_{12}\left(r_{1}\right) \widetilde{d}_{12}\left(r_{2}\right)\right]+\left[\widetilde{d}_{21}\left(r_{1}\right) \widetilde{d}_{21}\left(r_{2}\right)-\widetilde{d}_{22}\left(r_{1}\right) \widetilde{d}_{22}\left(r_{2}\right)\right]\right\}^{2}}{I\left(r_{1}\right) I\left(r_{2}\right)}
$$

were

$$
\left\{\begin{array}{l}
\widetilde{d}_{11}(r)=\cos ^{2} \rho(r)+\sin ^{2} \rho(r) \cos \delta(r), \\
\widetilde{d}_{12,21}(r)=\cos \rho(r) \sin \rho(r)(1-\cos \delta(r)), \\
\widetilde{d}_{22}(r)=\sin ^{2} \rho(r)+\cos ^{2} \rho(r) \cos \delta(r) .
\end{array}\right.
$$

Let us analyze the relationship of CDMA $\widetilde{W}\left(r_{1}, r_{2}\right)$ of blood plasma layer with variations orientations $\rho_{1}\left(r_{1}\right), \rho_{2}\left(r_{2}\right)$ of optical axes and the phase shifts $\delta_{1}\left(r_{1}\right), \delta_{2}\left(r_{2}\right)$ that introduced by the protein crystals in the points $\left(r_{1}, r_{2}\right)$. Table 1 shows the main characteristic values of CDMA $\widetilde{W}\left(r_{1}, r_{2}\right)$ in two arbitrary points $\left(r_{1}, r_{2}\right)$ of blood plasma layer.

Table 1. Characteristic values of CDMA $\widetilde{W}\left(r_{1}, r_{2}\right)$ of blood plasma laser image.

\begin{tabular}{|c|c|c|}
\hline $\begin{array}{c}\rho\left(r_{1}\right), \rho\left(r_{2}\right) \\
(0 \div \pi)\end{array}$ & $\begin{array}{c}\delta\left(r_{1}\right)=\delta\left(r_{2}\right) \\
(0 \div \pi)\end{array}$ & $\widetilde{W}\left(r_{1}, r_{2}\right)$ \\
\hline$\rho\left(r_{1}\right)=\rho\left(r_{2}\right)$, \\
\cline { 2 - 3 } \begin{tabular}{c}
$\rho\left(r_{2}\right)=0,5 \pi+\rho\left(r_{2}\right)$ \\
\cline { 2 - 3 }
\end{tabular} & $0,5 \pi$ & 1,0 \\
\hline \multirow{3}{*}{$\begin{array}{c}\rho\left(r_{1}\right)=\rho\left(r_{2}\right), \\
\rho\left(r_{2}\right)=0,5 \pi+\rho\left(r_{2}\right)\end{array}$} & $\pi$ & 1,0 \\
\cline { 2 - 3 } & $\delta_{2}\left(r_{1}\right)=0,5 \pi+\delta_{1}\left(r_{2}\right)$ & $\widetilde{W}\left(r_{1}, r_{2}\right)$ \\
\cline { 2 - 3 } & $0,5 \pi$ & 0,5 \\
\hline \multirow{3}{*}{$\rho\left(r_{1}\right)=\rho\left(r_{2}\right)}$, \\
$\rho\left(r_{2}\right)=0,5 \pi+\rho\left(r_{2}\right)$ & $\pi$ & 0,5 \\
\cline { 2 - 3 } & $\delta_{2}\left(r_{1}\right)=\pi+\delta_{1}\left(r_{2}\right)$ & $\widetilde{W}\left(r_{1}, r_{2}\right)$ \\
\cline { 2 - 3 } & $\pi$ & 0 \\
\cline { 2 - 3 } & $1,5 \pi$ & 0 \\
\hline
\end{tabular}




\section{OPTICAL SCHEME AND EXPERIMENTAL MEASUREMENTS OF TWO-DIMENSIONAL CDMA DISTRIBUTIONS OF BLOOD PLASMA}

Experimental investigations were carried out in the classical polarimeter the main parts and elements of which are presented in figure $1^{17}$.

It was illuminated by collimated $\left(\varnothing=10^{4} \mu \mathrm{m}\right) \mathrm{He}-\mathrm{Ne}$ laser beam $(\lambda=0.6328 \mu \mathrm{m})$ with the power of $50 \mu \mathrm{W}$. Polarization illuminator (quarter-wavelength plates 3,5 and polarizer 4) formed the beam with arbitrary polarization azimuth $\left(0^{0} \leq \alpha_{0} \leq 180^{\circ}\right)$ and ellipticity $\left(0^{0} \leq \beta_{0} \leq 90^{\circ}\right)$. Polarization images of blood plasma by means of microobjective 7 (focal distance $-1.5 \mathrm{~cm}$, aperture -0.2 , magnification $-4 \mathrm{x}$ ) were projected into the plane of light-sensitive area of CCD camera (overall amount of pixels $-800 \times 600$, light sensitive area size - 4000x3000 $\mu \mathrm{m}$, deviation of photosensitive characteristics from linear no more then $15 \%$ ), which provided the range of measuring the structural elements of blood plasma with the resolution 2-2000 $\mathrm{mm}$. Maximal resolution verification $(2 \mu \mathrm{m})$ where performed using the stage micrometer (linear scale), which image was projected into the light sensitive area of CCD camera with the help of microobjective 7.

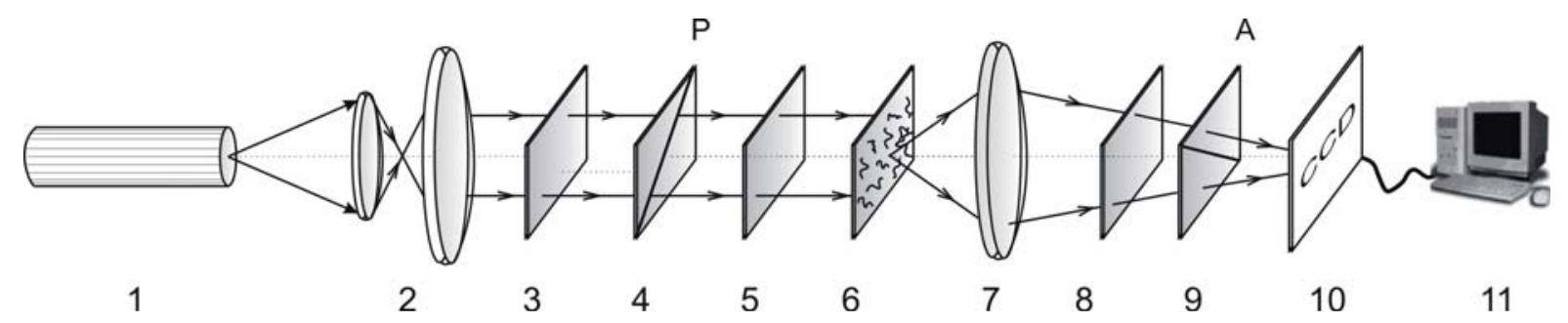

Figure 1. Optical scheme of polarimeter for measuring coordinate CDMA distributions of BT. Here $1-\mathrm{He}-\mathrm{Ne}$ laser $(\lambda=0.6328 \mu \mathrm{m})$; 2 - collimator; 3,5 and 8 - quarter-wave plates; 4 and 9 polarizers; 6 - BT histological section; 7 - projection microobjective; 10 CCD - camera; 11 - PC.

Minimal resolution ( $2000 \mu \mathrm{m}$ ) corresponds to the situation when the light sensitive area of CCD camera is entirely filled by two equal sized structural elements (light and dark) of stage micrometer. The conditions of the experiment were chosen in such a way that it enabled to reduce the space-angular aperture filtering while forming the blood plasma images.

Initially, according to the classical technique given $\mathrm{in}^{4}$, it was measured two-dimensional distribution $\left(\left(r_{m n}\right)-\right.$ coordinates of CCD camera pixels $(m \times n))$ of the real parts of Jones matrix elements $\widetilde{d}_{i k}(m \times n)$ of blood plasma layer.

Further rotation of the analyzer 9 transmission plane $(\Theta)$ in the limits $\Theta=0^{\circ} \div 180^{\circ}$ it is possible to determine minimum $\left(I_{\min }\left(r_{j k}\right)\right)$ and maximum $\left(I_{\max }\left(r_{j k}\right)\right)$ levels of intensity for each pixel $\left(r_{j k}\right)$ of CCD-camera 10 . Thus, the arrays of the intensity extreme values $I_{\min }(m \times n), I_{\max }(m \times n)$ of laser images of blood plasma 6 and the corresponding angles of rotation $\Theta_{\text {min }}(m \times n) \Leftrightarrow I_{\text {min }}(m \times n)$ of the analyzer 9 can be obtained.

On the basis of the obtained data it is possible to calculate the coordinate distribution of azimuth $\alpha(m \times n)$ and ellipticity $\beta(m \times n)$ of polarization and determine the two-dimensional distribution of phase shifts by using the following algorithms 


$$
\left\{\begin{array}{l}
\alpha(r)=0,5 \pi-\Theta(r), \\
\beta(r)=\operatorname{arctg} I_{\min }(r) / I_{\max }(r) \\
\delta(r)=\operatorname{arctg}\left[\frac{\operatorname{tg} 2 \beta(r)}{\operatorname{tg} \alpha(r)}\right] .
\end{array}\right.
$$

The value of CDMA $\widetilde{W}\left(r_{1}, r_{2}=r_{1}+\Delta r\right)$ of the two points $\left(r_{1}, r_{1}+\Delta r\right)$ shifted by the interval $\Delta r$ of the network of protein crystals is calculated using the algorithm (12), (13). Coordinate distribution $\tilde{W}(x, y)$ of blood plasma is determined by scanning with the $\Delta r=1$ pix step in two mutually transverse directions $(x=1 \div m, y=1 \div n)$.

\section{EXPERIMENTAL RESEARCHES OF TWO-DIMENSIONAL DISTRIBUTION OF CDMA OF BLOOD PLASMA WITH DIFFERENT PHYSIOLOGICAL STATES}

In our work we have performed a study of the relationship of statistical moments of the 1st - 4th order, which characterize the coordinate distribution $\widetilde{W}(x, y)$ of blood plasma of healthy patient and affected by appendicitis (acute and gangrenous appendicitis).

As objects of investigations (figure 2) were chosen the blood plasma layers (smears) of a three groups of patients healthy (figure 2a), affected by acute appendicitis (figure $2 \mathrm{~b}$ ) and affected by gangrenous appendicitis (figure 2c).

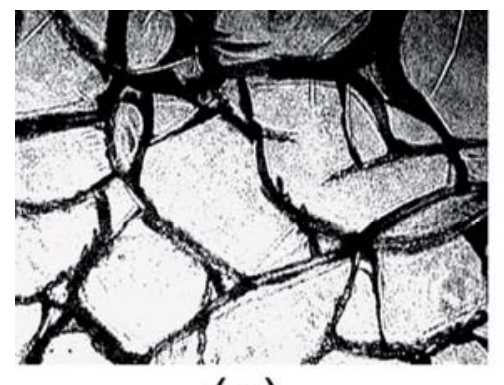

(a)

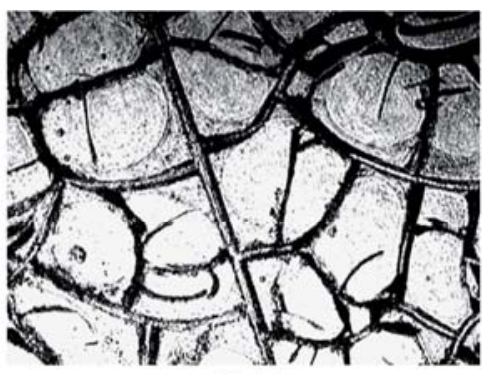

(b)

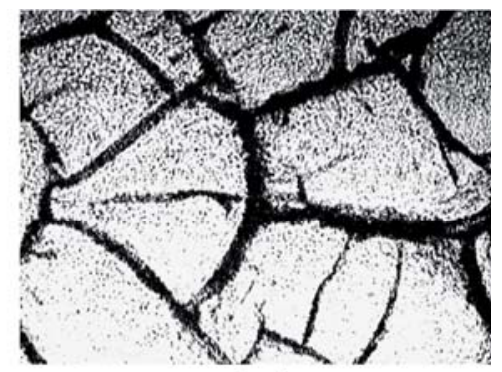

(c)

Figure 2. Polycrystalline networks of human blood plasma proteins. (a) - healthy patient; (b) - patient affected by acute appendicitis;

(c) - patient affected by gangrenous appendicitis.

It is well known fact that the concentration changes of optical-anisotropic blood plasma amino acids of albumin and globulin are connected with the deviation from the patient's normal physiological state. From optical point of view, such pathological processes can be revealed in structural optical-geometric changes of blood plasma polycrystalline networks: changes of optical axis directions of birefringent crystals; changes of phase shifting ability of polycrystalline networks.

Such transformations of optical-anisotropic network of blood plasma amino acids for all blood groups are illustrated in the figure 2. This laser images were obtained in crossed polarizer 4 and analyzer 9 (see figure 1).

The comparative qualitative analysis of laser blood plasma images (figure 2) did not reveal the sufficient changes in coordinate structure of amino acids polycrystalline network. This fact is additionally point out the topicality of objectification of such research. 
The series of coordinate distributions $(600$ pix $\times 800$ pix - fragments (a), (b), (c) and the histograms (fragments $(d)$, (e), (f)) of CDMA values $\widetilde{W}(x, y)$ of blood plasma layers all groups (group 1 - fragment (a), group 2 - fragment (b) and group 3 - fragment (c)) are presented on figure 3 .

The analysis of the obtained experimental data discovered that the coordinate distributions of CDMA (figure 3a,b,c) are formed by the set of the local areas (domains $\widetilde{W} \approx$ const ) with the maximum possible range of variation $0 \leq \tilde{W} \leq 1.0$ (figure $3 \mathrm{~d}, \mathrm{e}, \mathrm{f}$ ).

The experimental data satisfactorily correlate with the proposed model analysis of the blood plasma structure with the help of CDMA. Thus, there will always be points $(r, r+\Delta r)$ in the network of protein crystals that define such relationships between the parameters of anisotropy $(\rho, \delta)$ (table 1), which correspond to extreme values of CDMA $\widetilde{W}(r, r+\Delta r)=\left\{\begin{array}{l}0 ; \\ 1 .\end{array}\right.$

On the other hand, the specificity of the construction of blood plasma crystal network of various types (figure 2) is manifested in different probabilities of CDMA $\widetilde{W}(x, y)$ values.

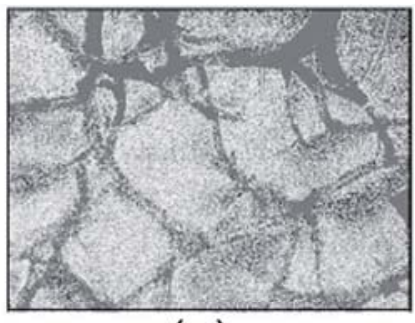

(a)

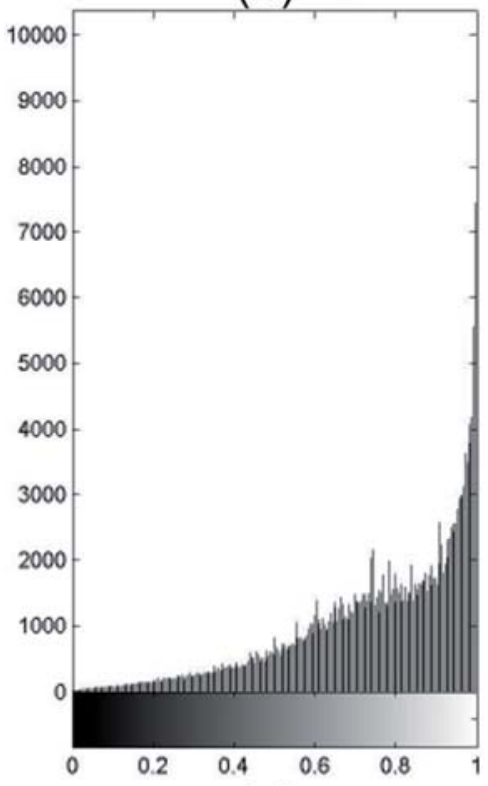

(d)

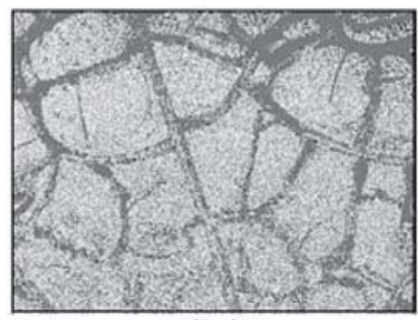

(b)

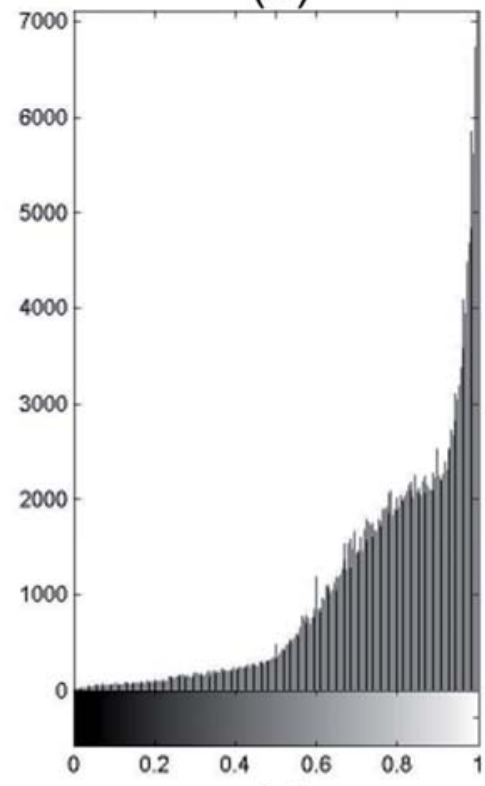

(e)

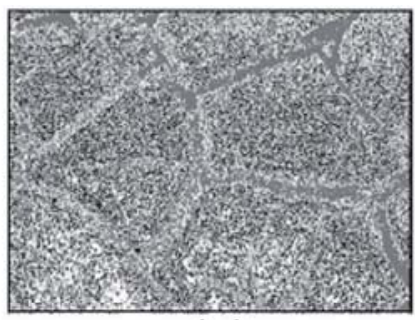

(c)

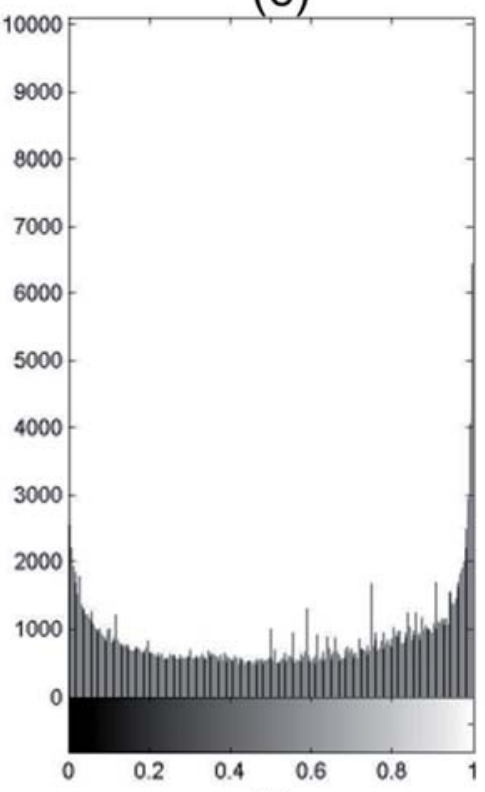

(f)

Figure 3. Coordinate distributions $(600$ pix $\times 800$ pix $)$ - fragments a, b, c; and the corresponding histograms - fragments $d$, e, f of the values $\widetilde{W}(x, y)$ of blood plasma laser images of all three groups. 


\section{STATISTICAL ANALYSIS OF TWO-DIMENSIONAL DISTRIBUTIONS OF BLOOD PLASMA CDMA}

With the aim of determination the ranges of statistical moments changes that characterize the coordinate distribution of $\widetilde{W}(x, y)$ and $\widetilde{V}(x, y)$ the following three groups of blood plasma samples were formed (healthy patients -21 samples; patients affected by acute appendicitis - 19 samples; patients affected by gangrenous appendicitis - 20 samples).

Statistical moments of the 1 st - 4 th-order $M_{k=1,2,3,4}$ of $Q=\left\{\begin{array}{l}\widetilde{V} \\ \widetilde{W}\end{array}\right.$ distributions were calculated with the help of Matlab software product on the basis of the following algorithms ${ }^{17}$

$$
M_{1}=\frac{1}{N} \sum_{i=1}^{N}\left|Q_{i}\right|, \quad M_{2}=\sqrt{\frac{1}{N} \sum_{i=1}^{N} Q_{i}^{2}}, \quad M_{3}=\frac{1}{M_{2}^{3}} \frac{1}{N} \sum_{i=1}^{N} Q_{i}^{3}, \quad M_{4}=\frac{1}{M_{2}^{4}} \frac{1}{N} \sum_{i=1}^{N} Q_{i}^{4} .
$$

were $N=m \times n$ - quantity of pixels of CCD-camera photosensitive area.

In Table 2 the results of calculations of the average $\left(M_{1}\right)$, the dispersion $\left(M_{2}\right)$, the skewness $\left(M_{3}\right)$ and the kurtosis $\left(M_{4}\right)$ of CDMA $\widetilde{W}(x, y)$ distributions of all groups of blood plasma layers are presented.

Table 2. Statistic moments of the $1^{\text {st }}-4^{\text {th }}$ orders of CDMA $\tilde{W}(x, y)$ of blood plasma layers.

\begin{tabular}{|c|c|c|c|}
\hline$M_{k}$ & $\begin{array}{c}\text { Group 1 } \\
(21 \text { samples })\end{array}$ & $\begin{array}{c}\text { Group 2 } \\
(19 \text { samples })\end{array}$ & $\begin{array}{c}\text { Group 3 } \\
(20 \text { samples })\end{array}$ \\
\hline$M_{1}$ & $0.78 \pm 0.089$ & $0.59 \pm 0.064$ & $0.43 \pm 0.043$ \\
\hline$M_{2}$ & $0.032 \pm 0.005$ & $0.046 \pm 0.005$ & $0.52 \pm 0.061$ \\
\hline$M_{3}$ & $2.1 \pm 0.22$ & $4.54 \pm 0.57$ & $6.4 \pm 0.78$ \\
\hline$M_{4}$ & $3.6 \pm 0.49$ & $6.7 \pm 0.68$ & $9.5 \pm 0.93$ \\
\hline
\end{tabular}

From the obtained data about the coordinate distributions of CDMA of blood plasma layers groups 1-3 one can see that:

- The average and the dispersion of distributions $\tilde{W}(x, y)$ differ insufficiently within $1.3\left(M_{1}\right)-1.5\left(M_{2}\right)$ times.

- The skewness values $M_{3}$ of distributions $\tilde{W}(x, y)$ of the investigated samples differ by 2.25 - 3 times; the kurtosis values - by $1.95-2.65$ times.

\section{CONCLUSION}

To characterize the degree of consistency of parameters of the optically uniaxial birefringent protein crystals nets of blood plasma a new parameter - complex degree of mutual anisotropy is suggested. The technique of polarization 
measuring the coordinate distributions of the complex degree of mutual anisotropy of blood plasma is developed. It is shown that statistic approach to the analysis of complex degree of mutual anisotropy distributions of blood plasma of various physiological states appears to be sensitive and efficient in differentiation of physiological state.

\section{REFERENCES}

[1] Patterson, M.S., Andersson-Engels, S., Wilson, B.C. and Osei, E.K., "Absorption spectroscopy in tissue-simulating materials: a theoretical and experimental study of photon paths," Appl. Opt. 34, 22-30 (1995).

[2] Alfano, R. R. and Fujimoto, J. G., eds., [Advances in Optical Imaging and Photon Migration, Vol. 2 of Topics in Optics and Photonics Series], Optical Society of America, Washington, D. C. (1996).

[3] van de Hulst, H. C., [Light Scattering by Small Particles], Dover, New York (1981).

[4] Morgan, S.P., Khong, M.P. and Somekh, M.G., "Effects of polarization state and scatterer concentration on optical imaging through scattering media," Appl. Opt. 36, 1560-1565 (1997).

[5] Demos, S.G. and Alfano, R.R., "Optical polarization imaging," Appl. Opt. 36, 150-155 (1997).

[6] Jacques, S.L., Roman, J.R., Lee, K., "Imaging superficial tissues with polarized light," Lasers in Surg. and Med. 26, 119-129 (2000).

[7] Born, M. and Wolf, E., [Principles of Optics], Cambridge U. Press, Cambridge, England (1999).

[8] Gori, F., Santarsiero, M., Vicalvi, S., Borghi, R., Guattari, G., "Beam coherence-polarization matrix," Pure Appl. Opt. 7, 941-951 (1998).

[9] Wolf, E., "Unified theory of coherence and polarization of random electromagnetic beams," Phys. Lett. A. 312, $263-$ 267 (2003).

[10] Tervo, J., Setala, T. and Friberg, A., "Degree of coherence for electromagnetic fields," Opt. Express 11, 1137-1143 (2003).

[11] Movilla, J.M., Piquero, G., Martínez-Herrero, R. and Mejías, P.M., "Parametric characterization of non-uniformly polarized beams," Opt. Commun. 149, 230-234 (1998).

[12] Mujat, C. and Dogariu, A., "Statistics of partially coherent beams: a numerical analysis," J. Opt. Soc. Am. A. 21, 1000-1003 (2004).

[13] Gori, F., "Matrix treatment for partially polarized, partially coherent beams," Opt. Lett. 23, 241-243 (1998).

[14] Mujat, M. and Dogariu, A., "Polarimetric and spectral changes in random electromagnetic fields," Opt. Lett. 28, 2153-2155 (2003).

[15] Mujat, M., Dogariu, A. and Agarwal, G.S., "Interferometric measurement of the degree of polarization and control of the contrast of intensity fluctuations," Opt. Lett. 29, 1539-1541 (2004).

[16]Ellis, J. and Dogariu, A., "Complex degree of mutual polarization," Opt. Lett. 29, 536-538 (2004).

[17] Ushenko, Yu.A., Tomka, Yu.Ya. and Dubolazov, A.V., "Complex degree of mutual anisotropy of extracellular matrix of biological tissues," Optics and Spectroscopy 110, 814-819 (2011). 